\title{
ULTRASONIC GRAIN NOISE MODELLING APPLICATIONS TO TITANIUM ALLOY INSPECTION
}

\author{
THEODOR TRANCA $^{1} \&$ IULIANA RADU ${ }^{2}$ \\ ${ }^{1}$ Diac Servicii srl, Romania \\ ${ }^{2}$ Zirom-SA, Romania
}

\begin{abstract}
Ultrasonic non-destructive evaluation (NDE) relies on the scattering of waves from discontinuities, such as fractures or voids, to probe media otherwise invisible to the naked eye. Whilst this has been industrially exploited for several decades within acoustically transparent materials, many materials maintain a microstructure that causes scattering of the propagating waves. This undermines the aforementioned premise as it becomes exceedingly difficult to discern the features of interest from the scattering inherent to microstructural features, thereby limiting the range of materials which can be reliably inspected. Experimental investigations confirm the challenges and significant shortcomings for the inspection of future industrial components where such microstructures are desirable for their mechanical properties. It is demonstrated that the rapid increases in scattering with the frequency severely limit the achievable sensitivity of conventional ultrasound techniques. A review of the latest advances in ultrasound technology explore the opportunities to exceed current limitations and advance the capability of ultrasonic NDE.
\end{abstract}

Keywords: pulse volume, signal noise ratio, ultrasonic grain noise.

\section{INTRODUCTION}

Commercial Ti-6A1-4V forgings are widely used in the rotating components of aircraft engines. The failure of such parts can be quite catastrophic because of the large amount of kinetic energy involved. Ultrasonic (UT) inspection is one of the non-destructive evaluation (NDE) methods widely used by titanium forging manufacturers because of its capability to penetrate to the interior of a component. However, the detection of smaller defects is made difficult by issues such as the presence of a high level of noise signals backscattered from the two-phased $(\alpha+\beta)$ microstructure. Sometimes the backscattered noise level is comparable or even higher than the small defect response. The inspection sensitivity, a quantity that describes the smallest defect a system can detect, is directly related to the backscattered grain noise (Thompson and Margetan [1], Margetan et al. [2]). The UT inspection of the titanium forgings billets is presently performed using one of the automated UT testing methods. The billet volume is scanned by probes arranged perpendicular to the billet. The entry echo is monitored as a function check and the back-wall echo as well as any echo from a possible defect. This paper proposes to analyse the typical problem of UT grain noise related to this kind of inspection.

\section{DAC COMPENSATION}

Electronic distance-amplitude correction (DAC) is applied in order to equalize the signal's amplitude of the reflector, regardless of its distance from the source (Fig. 1).

Increased gain is required with depth because the UT beam attenuates with distance and diverges beyond the focus position that is placed near the surface. A higher gain applied with depth has the effect of increasing the UT noise with depth. In some cases, where material thickness exceeds $50 \mathrm{~mm}$ or so, the noise becomes too large because so much gain has to be applied. In such cases, a second zone inspection is applied, whereby the transducer beam focus is moved further into the part, either by moving the transducer closer to the surface or 
by using a different transducer that has different focusing characteristics. A second scan is then made with an inspection gate on the deeper region.

\section{UT GRAIN NOISE}

UT grain noise is present in forging inspections and can have the effect of masking the echoes from small or subtle defects in the metal. The term "grain noise" is generally associated with the root mean square (RMS) signal attributable to microstructure. As indicated in Fig. 2(d), this noise arises from the scattering of sound waves by the metal microstructure. Ti6-4 forgings exhibit an internal structure on several length scales:

- Individual micro-grains - that is, single crystals of metal with atoms arranged in a regular lattice - are presented on the smallest scale (Fig. 2(a)).

- Neighbouring micro-grains with aligned or partially aligned lattices can form larger entities, such as platelets, colonies, or macro-grains having large dimensions compared to the incident sonic wavelength (Fig. 2(b)).

- The largest structures, macro-grains, are often visible without magnification when polished metal surfaces are properly etched - for example, in flow-line photos like those shown in Fig. 2(c).

The microstructure influences UT pulse/echo inspections in three basic ways:

- The attenuation of sound energy acts to decrease the amplitude of echoes from possible defects;

- Backscattered noise from grain boundaries acts to mask echoes from small or subtle flaws; and

- Velocity inhomogeneity cause distortions of propagating sound beams, which lead to fluctuations in echo amplitudes.

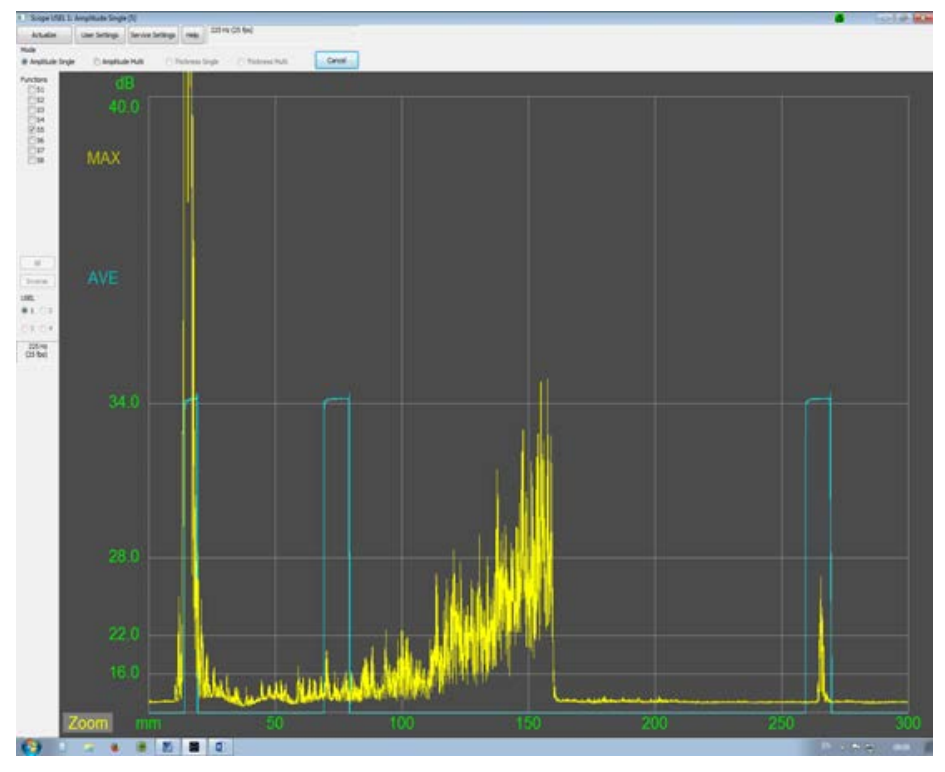

Figure 1: Distance-amplitude correction. 


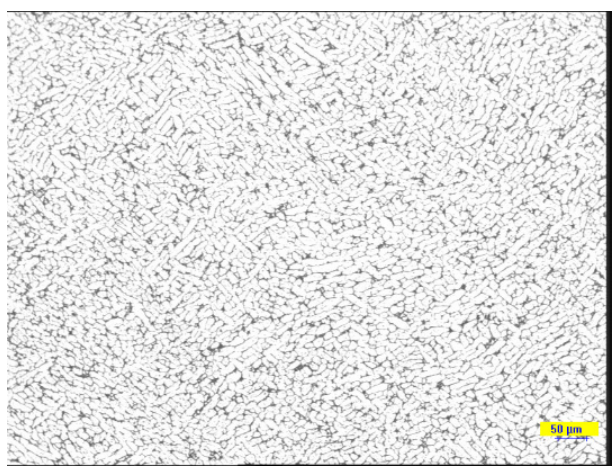

(a)

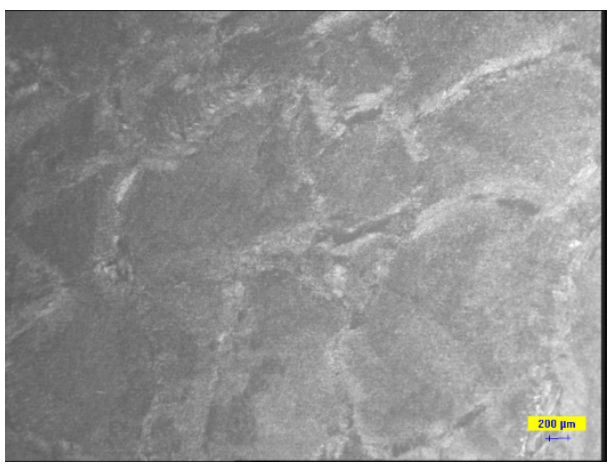

(c)

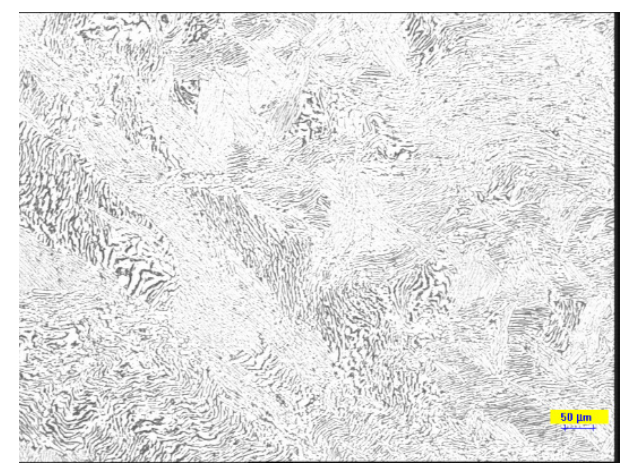

(b)

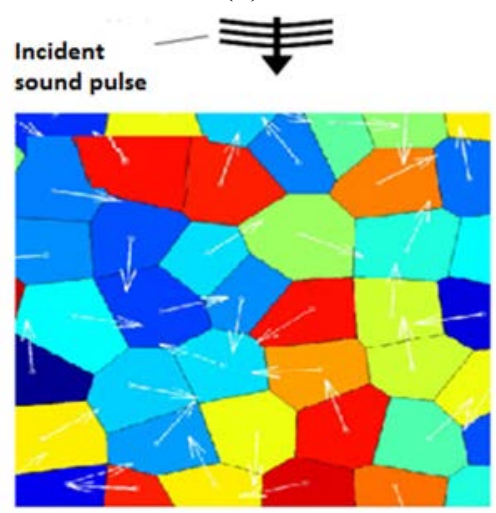

(d)

Figure 2: Scattering of sound waves by microstructural boundaries results in grain noise.

Ultrasound is fundamentally limited by the presence of scattering once the wavelength becomes dimensionally similar to the characteristic dimension of the in-homogeneity of the propagation medium. This ultimately limits the range of materials which can be reliably inspected and restricts the maximum resolution which can be achieved. The appearance of the grain noise during a UT inspection is presented in Fig. 3. Scattering occurs within polycrystalline materials due to a contrast in phase velocity at each grain boundary of the microstructure.

As the coherent wave is scattered, it becomes very difficult to discern the signals of interest, stemming from defects such as voids and fractures, from those inherent to the microstructural features of the material.

Additional difficulty can arise due to macro-anisotropy. Anisotropic materials have properties that are dependent on direction. Metallic materials such as titanium alloys can also exhibit stiffness variations on a macro-scale. When this is the case, this can present challenges for a UT inspection as it affects wave propagation and causes deviations from the equivalent straight-ray path for an isotropic medium. The effect of anisotropy is analogous to a defocusing and skewing of the beam, which rapidly reduces measured signal amplitudes. The total backscattering will be controlled by grain morphology, grain orientation and elastic 
anisotropy of the microstructure. Such noise variations are believed to be correlated with the local microstructural variations that arise from thermo-mechanical processing (TMP).

The grain noise appears as a mottled pattern whose average amplitude varies along the scanning surface, reflecting changes in the properties of the microstructure. This reduced the ability to distinguish signals from noise, often quantified by the signal-to-noise ratio (SNR).

A UT "C"-scan image is created by displaying the largest peak-to-peak or rectified peak amplitude that is seen within the gate during a two-dimensional scan of the transducer above the forging. Such a "C"-scan is shown in Fig. 4.

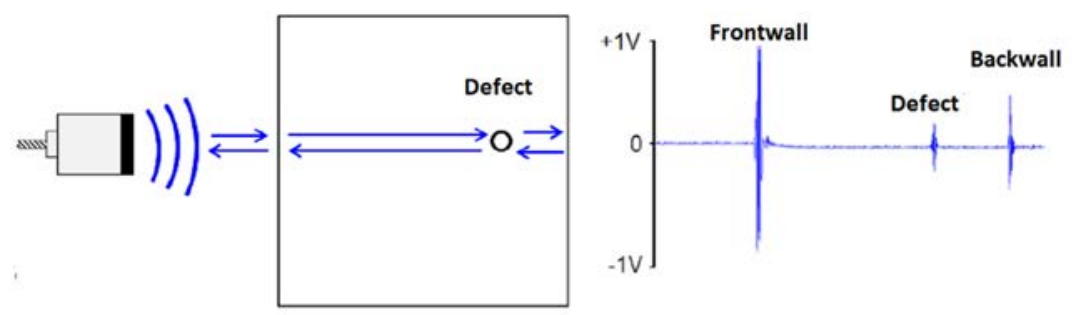

(a)

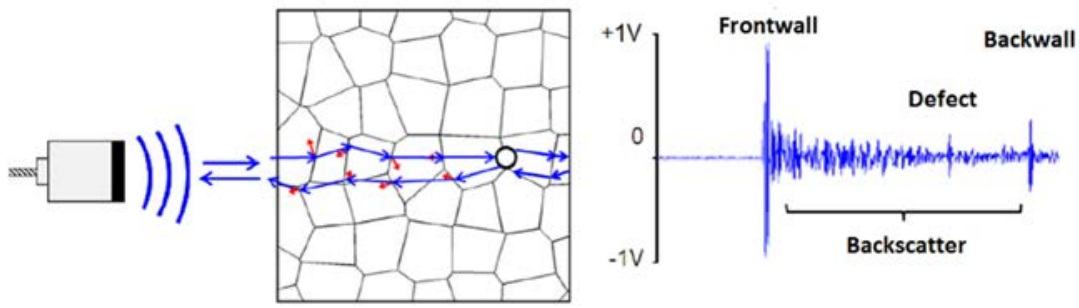

(b)

Figure 3: Schematic of a pulse-echo ultrasonic immersion inspection of a cylindrical defect within (a) an ultrasonically clean material; and (b) a highly scattering material. (Source: Van Pamel [3].)

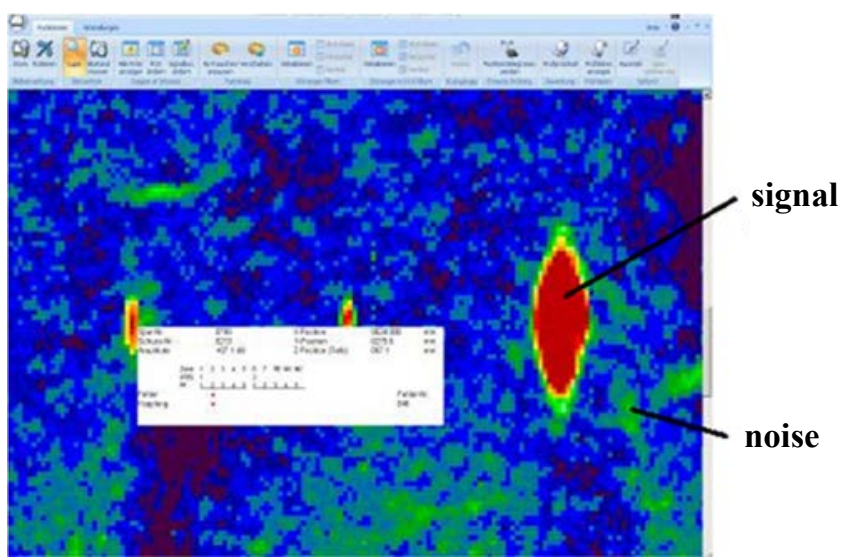

Figure 4: UT “C”-scan containing an indication in the presence of noise. 


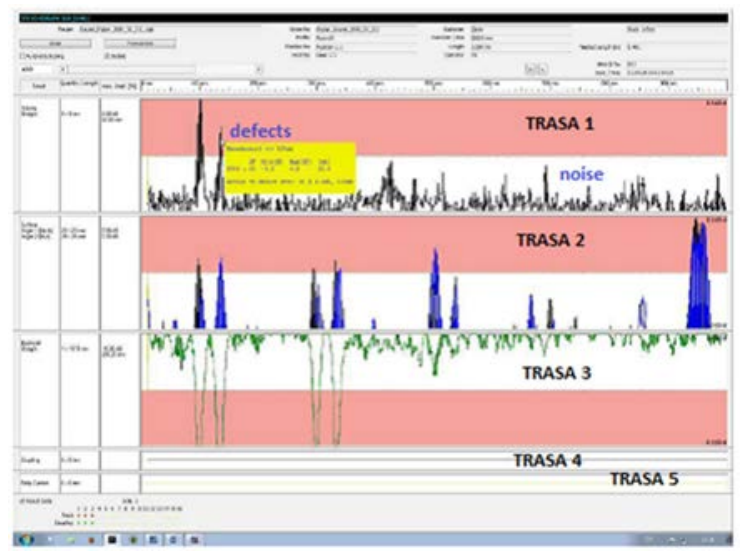

Figure 5: UT "B"-scan containing indications of the defects and the grain noise. TRASA 1: record of the normal transducer (S) scanning; TRASA 2: record of the angled transducers (A1 and A2) scanning; TRASA 3: record of the back-wall echo evolution (normal transducer S); TRASA 4: record of the coupling evolution (S, A1, A2); TRASA 5: record of the eddy-current examination.

Fig. 5 presents a "B"-scan of a forging Ti6-4 cylindrical reference block containing two artificial reflectors (\#2 FBH and \#3 FBH) positioned in the centre of the billet. The scanning was performed using an AUT system, according to the Helical Testing Method. The grain noise is visible along the whole billet examination, having a shape depending on the transducer frequency and the depth of the defect.

\section{SIGNAL/NOISE REPORT}

The severity of grain noise is usually quantified using a so-called SNR. The competing grain noise in the vicinity of the reflector can be analysed to determine various attributes, such as the average noise amplitude and the peak noise amplitude. The SNR quantifies the relative sizes of the reflector signal and the competing noise

$$
\frac{S}{N}=\frac{\text { peak defect amplitude }}{\text { peak noise amplitude }} .
$$

If a metal defect is small in lateral extent and if its SNR is too low - for example, near or below unity - then it is unlikely to be detected by examining a "C"-scan image. Ensuring adequate SNR for critical defects is, thus, of key importance when designing a UT inspection protocol.

To predict the noise levels, we need detailed knowledge of the metal microstructure which enters the model calculations through certain frequency-dependent factors known as "backscatter coefficients" or "Figures-of-Merit" (FOM). The resulting expression for "noise" produced under these conditions contains a factor which depends on the volumetric density of scatters and their RMS scattering amplitude in the backscattered direction. FOM appears to be a material property that is useful in characterizing microstructures.

Such a FOM can be directly related to the time-domain RMS noise observed in an A-scan. For example, for a given inspection configuration, a material having a FOM of 2 produces an A-scan with noise which is double that of a material with a FOM of unity. 
A summarized eqn (2) is shown here:

$$
N r m \mathrm{~s} \sim F O M C_{1} \iiint_{-\infty}^{\infty} C^{4}(x, \mathrm{y}, \mathrm{z}) d x d y d z,
$$

where:

$C 1$ represents the variables associated with the inspection configuration; and the volume integral of $C$ represents the incident beam.

In the theories of Rose, Han and Thompson, under the assumption that the solid density is independent of position, $\operatorname{FOM}(\omega)$ and $\eta(\omega)$ for longitudinal waves propagating in the S3 direction are related to microstructure features by eqn (3):

$$
F O M(\omega)^{2}=\eta(\omega)=\frac{K^{4}}{\left(4 \pi \rho V_{l}^{2}\right)^{2}} \int d^{3} \vec{S}\left\langle\delta \mathrm{C}_{33}(\vec{r}) \delta \mathrm{C}_{33}\left(\overrightarrow{r^{\prime}}\right)\right\rangle e^{2 i k S_{3}}
$$

where:

$\eta(\omega)$ is the backscattering power coefficient;

$\omega$ is the angular frequency;

$\mathrm{k}$ is the magnitude of the wave vector of the incident wave;

$\rho$ is the density of the solid;

$\mathrm{Vl}$ is the longitudinal wave velocity;

$\mathrm{S}=\vec{r}-\overrightarrow{r^{\prime}}$ is a vector defined by the two points $\vec{r}$ and $\overrightarrow{r^{\prime}}$ in the solid medium;

$\mathrm{S} 3$ is the component of $\vec{S}$ in the direction of wave propagation (3-direction);

$\delta \mathrm{C} 33(\vec{r})$ is the local deviation of the elastic constant from its Voigt average;

$\delta \mathrm{C} 33(\vec{r})=(\mathrm{C} 33-\mathrm{C} 33$ Voigt $)$ and the notation $\langle\ldots\rangle$ denotes an ensemble average; and

$\left\langle\delta \mathrm{C} 33(\vec{r}) \delta \mathrm{C} 33\left(\overrightarrow{r^{\prime}}\right)\right\rangle$ is known as the two-point correlation of elastic constants, which

describes the correlation in the perturbation in the elastic stiffness.

Despite having developed experimental methods to measure FOM, there were no means to predict it from the known material properties. For a typical industrial inspection of a billet or rectangular forging, such FOM information is not generally available, although it could be deduced by analysing backscattered noise waveforms from regions where the microstructure is spatially uniform ( $\mathrm{Yu}[4])$.

\section{PULSE VOLUME}

In many cases of practical interest, the SNR for a given reflector embedded in a particular microstructure can be related to the volume of the UT pulse that is incident on the reflector. A UT transducer can be regarded as injecting a sonic pulse into the metal component under inspection. As we see in Fig. 6, this pulse changes its shape as it propagates due to beam focusing and diffraction. The pulse will have a volume of space determined by its length in the propagation direction and its cross-sectional area, at the depth where it is determined. The pulse volume will tend to be smallest in the focal zone where the beam intensity is largest (Fig. 6(a)). When the pulse impinges on a reflector, sonic echoes are reflected back toward the transducer by the reflector itself and by the metal grains that surround it. If the reflector is smaller than the beam cross-section and the pulse volume encompasses many grains, then it can be argued that the SNR will increase as the pulse volume decreases. This is because:

1. The reflectivity of a given grain compared to that of the embedded reflector is relatively independent of the pulse volume if both are hit by the pulse; and 
2. When the pulse volume is reduced, fewer grains are excited, which can produce echoes arriving at the same time as the echo from the reflector (Fig. 6(b)).

Experimentally, the pulse volume for each transducer in its focal zone was determined in two steps:

1. Measuring the time duration of a back-wall echo and translating that into a pulse length.

2. Measuring the lateral area of the focal spot. The latter was done by scanning the focal area of the transducer over an FBH positioned in the reference block and analysing the resulting images.

For these determinations, a line-focused $5 \mathrm{MHz}, 3 / 4$ " crystal diameter and $200 \mathrm{~mm}$ water focal distance immersion transducer was used, together with two reference blocks made from Ti6-4. The spot size was determined by scanning the transducer with many \#3 FBH, positioned at increasing depths in the material, and then analysing the resulting A-scan images. This scanning was made in real examination conditions, by respectively moving the reference block in a circular direction and the transducer in feed directions (Fig. 7). The values of the lateral extensions determined for two different diameters billets ( $\Phi$ 111.5 and $\Phi 265 \mathrm{~mm}$ ), in both scanning directions and at different depths of the targeted FBH, are shown in Fig. 8.

The shape of the entry surface influences the focusing of the sonic beam within the forging, and, hence, affects both the amplitude of defect echoes and the level of competing grain noise (Fig. 9).

The effects of surface curvature are visible when the two reference blocks (ø $111.5 \mathrm{~mm}$ and $\varnothing 265.0 \mathrm{~mm}$ ) are scanned in order to determine the values of the lateral extensions (Fig. 8) and the gain correction (Fig. 10), for each artificial defect of these.

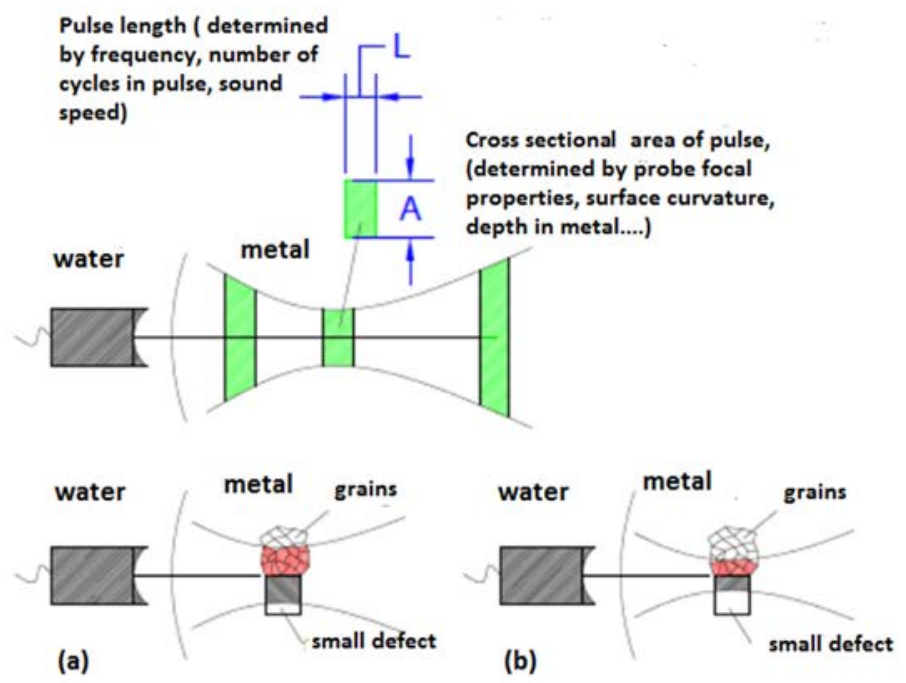

Figure 6: (a) As a sonic pulse propagates (from left to right), the volume that it occupies varies; and (b) a smaller pulse volume interacts with fewer grains near a defect, usually leading to a higher SNR. 


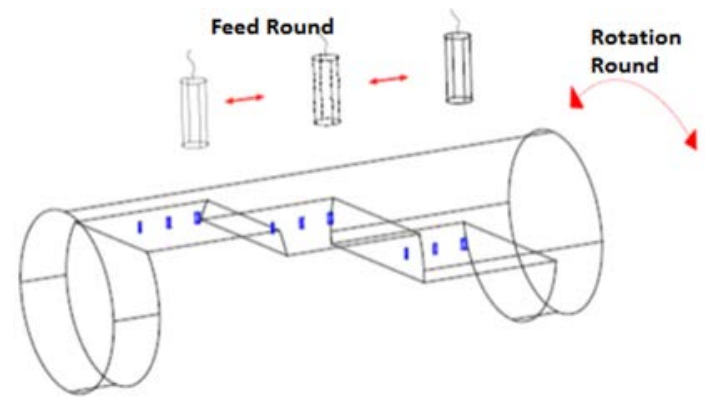

Figure 7: Scanning of the reference block in order to determine the lateral extensions at different depths.
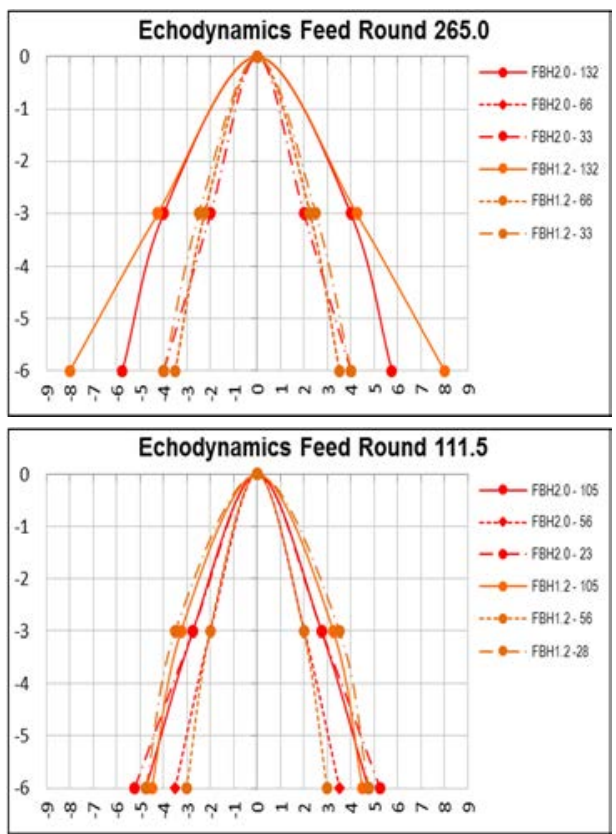
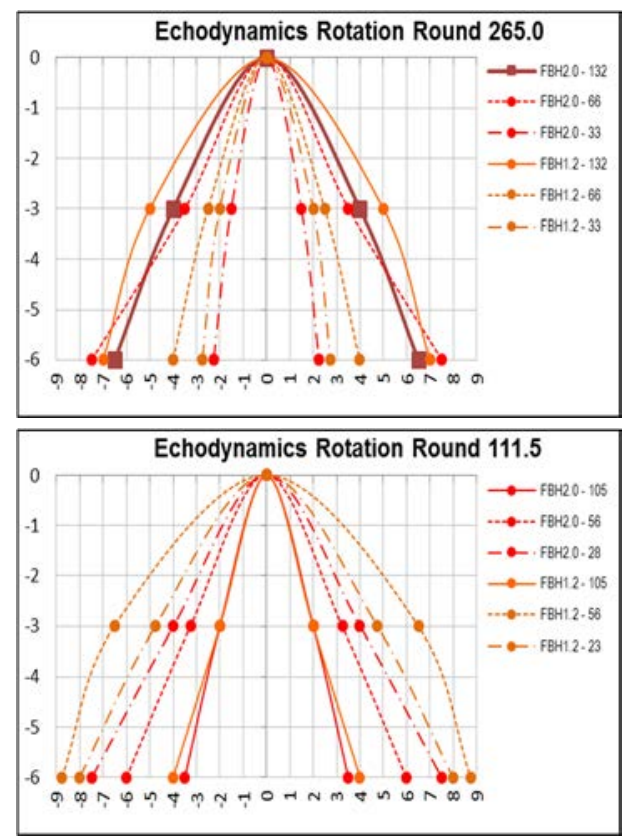

Figure 8: Lateral extensions measured on the reference blocks.

The difference in diameter leads to significant changes in the examination settings, even though the same transducer is used for each measurement, positioned at a fixed water path $(90 \mathrm{~mm})$ and the materials of the two blocks have the same (identical) chemical composition, heat treatment and UT transparency.

Both the lateral extension and the gain correction curve are strongly affected by the value of the billet radius.

\section{SNR RELATING TO THE PULSE VOLUME}

A mathematical expression empirically established between the pulse volume and SNR could be developed by making a series of reasonable approximations to the full model formalism. That rule of thumb is summarized in eqns (4) and (5) (Margetan et al. [6]). 

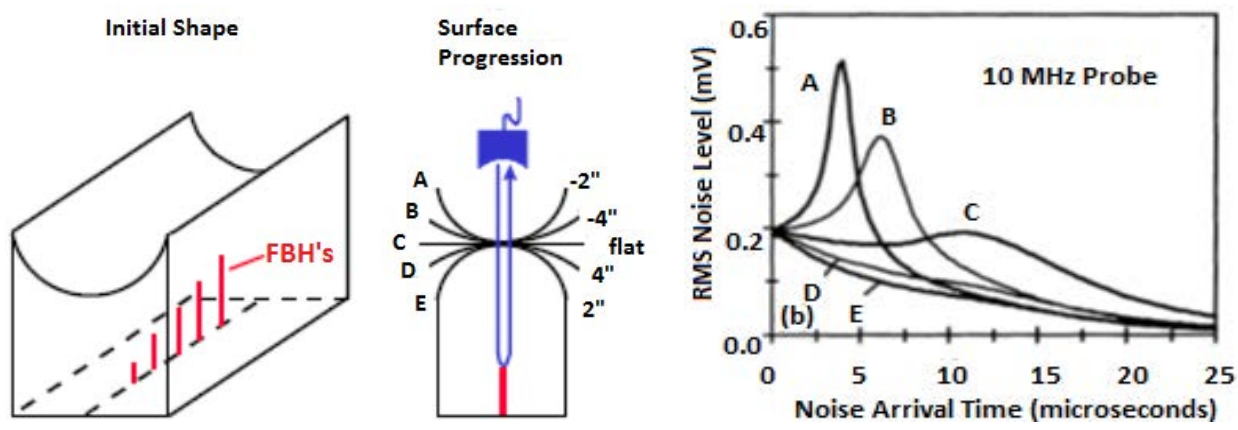

Figure 9: The effect of the shape of the entry surface. (Source: Margetan et al. [5].)
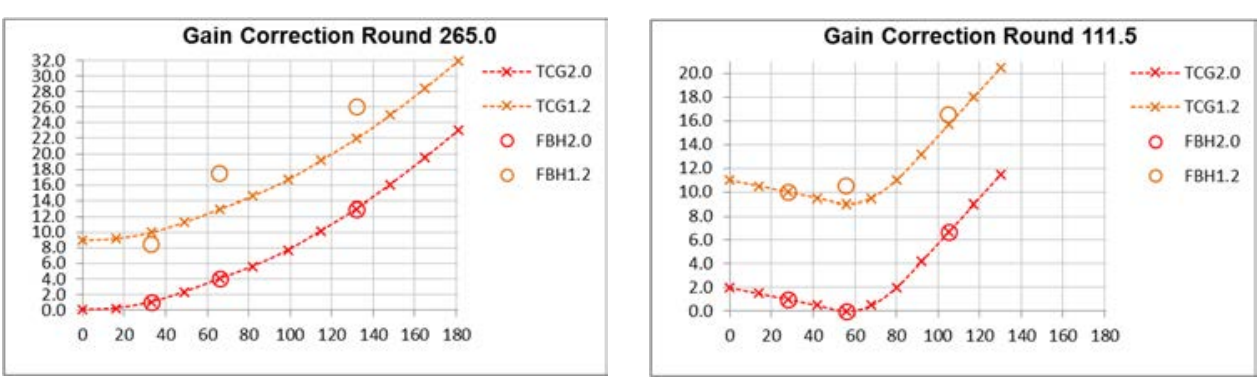

Figure 10: The influence of the billet radius over the gain correction curve.

It essentially says that for a fixed inspection frequency, SNR is proportional to $1 /$ the square root of the sonic pulse volume at the flaw depth. The constant of proportionality depends on the inherent noisiness of the microstructure, as quantified by the so-called FOM value and on the reflectivity of the defect, as quantified by the scattering amplitude $\left(A_{\text {flaw }}\right)$. The rule is specific for signal-to-noise computed using the peak (on-axis) defect amplitude and the so-called RMS average grain noise level. Consider the case of a small flaw located somewhere along the beam's central ray (but not necessarily at the focus). Using "Gaussian Beam" and "tone-burst" approximations, the ratio of flaw signal amplitude to "localized RMS grain noise" is approximately given by eqn (4):

$$
\frac{S}{N}=\sqrt{\frac{16}{\pi v_{\text {metal }} W x W y \Delta t}} \frac{A_{\text {flaw }}(f 0)}{F O M(f 0)},
$$

where:

- Wx and Wy are the lateral beam widths at flaw depth;

- $\Delta \mathrm{t}$ is the pulse duration;

- FOM is the noise FOM at centre frequency; and

- $A_{\text {flaw }}$ is the flaw scattering amplitude at centre frequency. 
Thus, for any given measure of noise amplitude, even if pulse centre frequency changes,

$$
\frac{S}{N} \propto \frac{1}{\sqrt{\begin{array}{c}
\text { volume"of the ultrasonic pulse } \\
\text { in vicinity of the defect }
\end{array}}}
$$

so long as:

- Flaw is small compared to beam diameter;

- Microstructure is relatively uniform within scan region;

- Pulse volume contains "many" grains ( $>5$ grains);

- Grain noise FOM and flaw scattering amplitude $\mathrm{A}_{\text {flaw }}$ increase with frequency at same rate.

This empirical relation is specifically for signal-to-noise computed using the peak (on-axis) defect amplitude and the so-called RMS average grain noise level. If the probe centre frequency changes, it is likely that the pulse volume, flaw scattering amplitude and microstructural FOM will also change.

However, for a small FBH defect in titanium alloys, both the FOM and the flaw scattering amplitude are approximately proportional to frequency. Thus, $\mathrm{A}_{\text {flaw }} / \mathrm{FOM}$ is approximately independent of frequency and it can be argued that the SNR remains roughly proportional to $1 /$ square root of pulse volume, even for situations where the frequency changes (so long as the microstructure and defect particulars remain fixed).

The eqn (4) also predicts the SNR to change according to the change in ratio between $\mathrm{A}_{\text {flaw }}$ and FOM with frequency. This led to the introduction of multi-zone transducers and the configuration of transducers, in which each focus at a different depth within the billet, to improve overall SNR.

The whole billet volume is zoned into several inspection zones with different depths, as shown in Fig. 11.

\section{THE INSPECTION IMPROVEMENT}

We analysed two examples of references blocks scanned on the AUT system. The main UT characteristics of the blocks are presented in Figs 8 and 10. The pulse volumes are significantly different for these two trials because in each case, the inspection gain was set so that the response from the \#3 FBH reference hole would be at $60 \%$ full screen height (FSH). The peak noise amplitude in the area surrounding the FBH array was measured at that gain setting. It is observed that the FBH amplitudes in each of Fig. 12 and Fig. 13 are essentially equal, but the mean and peak noise levels are clearly higher for the weaker focusing case. This directly illustrates the improvement in SNR that occurs when the sonic pulse volume is reduced. The focusing will be different for each case, even the same linefocused transducer, positioned at the same water path was used for both examinations. The reason for this effect is the significant difference between the radius of the two billets. This difference is properly highlighted in Fig. 8 that presents the lateral extensions of the sound beam measured on two different blocks (respectively Ø 111.5 and $\varnothing 265 \mathrm{~mm}$ ). Comparing the diagrams, it is obvious that, at the same deep and for same artificial defect (\#3 FBH), the lateral extension is bigger for the minor diameter at the same depth and for same artificial defect (\#3 FBH). As a result, the pulse volume will be reduced for the examination of the $\varnothing 265$ block and increased for the Ø111.5 block. 

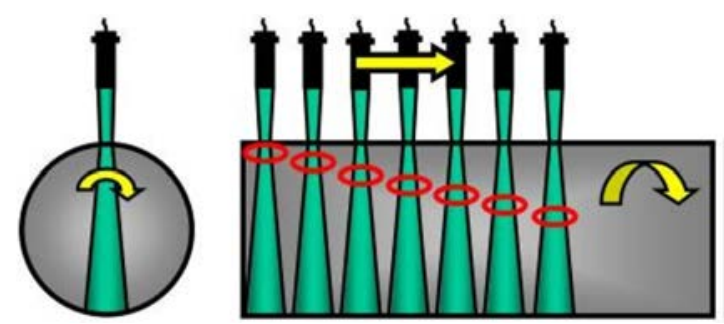

Figure 11: Testing principle of the rotational multi-zone inspection with highly focused probes (example: seven depth zones are covered by seven straight-beam probes). (Source: Deutsch and Joswig [7].)
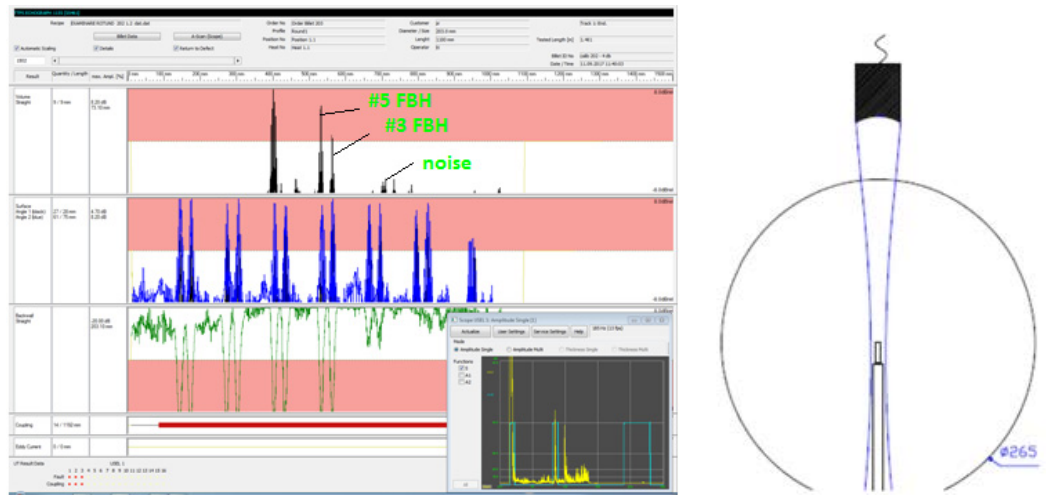

Figure 12: Stronger focusing transducer: FBH located at the focal plane; \#3 FBH at $60 \%$ FSH (\#5 FBH at 130\% FSH); peak noise amplitude at 12\% FSH; SNR 5:1.
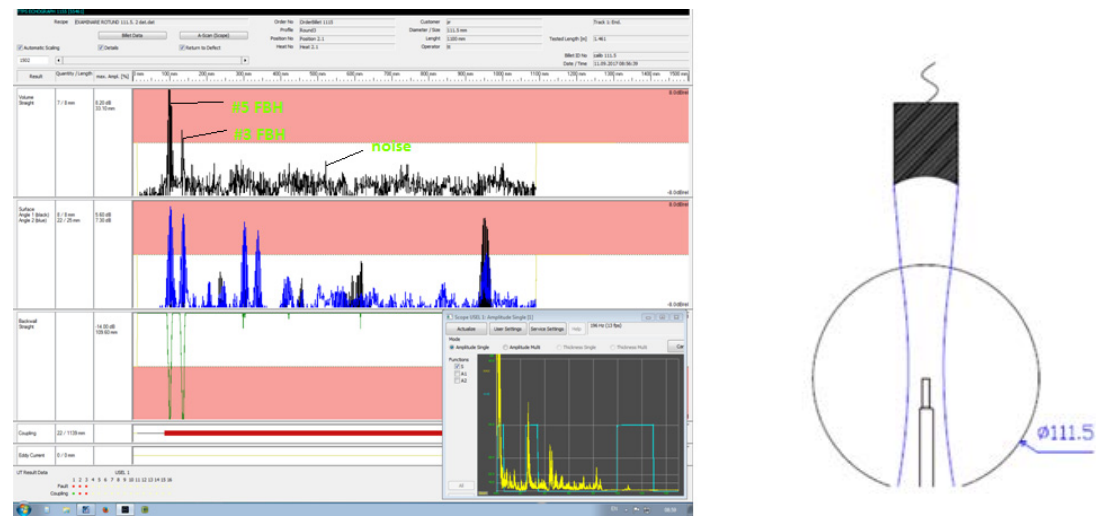

Figure 13: Weak focused transducer: FBH located beyond the focal plane; \#3 FBH at $60 \%$ FSH (\#5 FBH at 130\% FSH); peak noise amplitude at 33\% FSH; SNR 2:1. 


\section{SUMMARY}

Enhancing the UT inspection of highly scattering materials is required to improve the capability to both detect and characterise - including classification and sizing - sub-surface flaws within coarse-grained, polycrystalline materials. Since the ability to characterise these materials is governed by resolution, meeting this purpose rests on enabling inspections at the highest possible frequencies, despite the consequent increase in scattering, in order to maximise resolution. In industrial inspections of titanium billets and flat-faced materials, the FOM values describing the contribution of the microstructure to backscattered grain noise are not usually available. Without such FOM information, it is not possible to make accurate predictions of absolute noise characteristics.

However, these noise models can be used to predict the change in level of SNR that is likely to occur when the microstructure is fixed, but some features of the inspection are changed. By using these models and measuring the material noise, we can anticipate the behaviour modifications of the UT system when few of the main variables of the examination are modified (transducer characteristics such as diameter, focal length, frequency, or the billet diameter size, leading to pulse volume modification).

\section{REFERENCES}

[1] Thompson, R.B. \& Margetan, F.J., Use of elastodynamic theories in the stochastic description of the effect of microstructure on ultrasonic flaw and noise signals. Wave Motion, 36(2002), pp. 347-365.

[2] Margetan, F.J., Han, K.Y., Yalda, I., Goettsch, S. \& Thompson, R.B., The practical application of grain noise models in titanium billet and forgings. Review of Progress in QNDE, 14B, p. 2129.

[3] Van Pamel, A., Ultrasonic Inspection of Highly Scattering Materials, Department of Mechanical Engineering, Imperial College London, October 2015.

[4] Yu, L., Understanding and Improving Ultrasonic Inspection of Jet-Engine Titanium Alloy, Iowa State University, 2004.

[5] Margetan, F.J., Umbach, J., Roberts, R., Friedl, J. \& Degtyar, R., Inspection Development for Titanium Forgings - DOT/FAA/AR-05/46, Air Traffic Organization Operations Planning Office of Aviation Research and Development: Washington, DC, 20591, May 2007.

[6] Margetan, F.J., Yalda, I. \& Thompson, R.B., Ultrasonic grain noise modeling: Recent applications to engine titanium inspections. Review of Progress in Quantitative Nondestructive Evaluation, 16, 1997.

[7] Deutsch, W.A.K. \& Joswig, M., Automated ultrasonic testing - systems for bars and tubes. Presented at 11th European Conference on Non-Destructive Testing (ECNDT 2014), 6-10 October, Prague, Czech Republic, 2014. 